\title{
LATTICES AND THE ADJOINT GROUP OF A LIE GROUP
}

\author{
BY \\ HOWARD GARLAND(1) AND MORIKUNI GOTO(2)
}

1. Introduction. By an analytic group and by an analytic subgroup of a Lie group, we mean a connected Lie group and a connected Lie subgroup of a Lie group, respectively. Unless specified otherwise, an analytic subgroup and its corresponding Lie subalgebra will be denoted by the same capital Roman and capital German letter, respectively. For example, if $G$ denotes an analytic group and $A$ denotes an analytic subgroup of $G$, then $(5$ will denote the Lie algebra of $G$, and $\mathfrak{A}$ will denote the subalgebra of $\mathfrak{G}$ corresponding to $A$. We make the convention that the Lie algebra of a Lie group is the tangent space of that group at the identity.

Let $G$ be an analytic group, and let $A(G)$ denote the group of all continuous automorphisms of $G$. We give $A(G)$ the compact-open topology. Let GL(5) be the Lie group of all linear automorphisms of the vector space $\mathfrak{G}$, and let $A(\mathfrak{G})$ denote the closed subgroup of all Lie algebra automorphisms of $\mathbb{5}$; then $A(G)$ can be naturally imbedded in $A(\mathfrak{G})$ as a closed subgroup (see [2, p. 138]). When $G$ is simply connected, $A(G)$ and $A((5)$ coincide. We let $I(G)$ denote the analytic subgroup of $A(G)$ consisting of all inner automorphisms of $G$. $I(G)$ depends only on $(\mathfrak{b}$, and therefore will also be denoted by $I(\mathfrak{b}) ; I(G)$ will be called the adjoint group of $G$ (or of (5). We identify $g l(\mathfrak{5})$, the Lie algebra of $G L(\mathfrak{F})$, with the Lie algebra of all endomorphisms of $(\mathfrak{5}$; then $A(\mathfrak{5})$ corresponds to the subalgebra $a(\mathfrak{5})$, consisting of all derivations of $(\mathfrak{5} ; I(\mathfrak{G})$, on the other hand, corresponds to the subalgebra $i(\mathfrak{G})$ of all inner derivations of $\mathfrak{G}$.

If $S$ is a subset of $G$, we set

$$
\Delta(S)=\{\tau \in A(G) \mid \tau(s)=s, \text { for all } s \in S\}
$$

then $\Delta(S)$ is a closed subgroup of $A(G)$, and we let $\Delta^{0}(S)$ denote the identity component of $\Delta(S)$.

Definition. A discrete subgroup $\Gamma$ of a Lie group $L$ is called a lattice in case a measure $\mu$, induced on the coset space $L / \Gamma$ by a right invariant Haar measure

Received by the editors March 9, 1966.

(1) Research partially supported by the Army Research Office, Durham.

(2) Research partially supported by NSF GP-1928. 
on $L$, satisfies $\mu(L / \Gamma)<\infty$. If, moreover, $L / \Gamma$ is compact, then $\Gamma$ is called a uniform lattice.

It is not difficult to see that a uniform lattice is a lattice, and that if $L$ contains a lattice, then $L$ is unimodular. A lattice in a solvable, analytic group is always a uniform lattice (see [13]). It is our purpose here to prove the following two theorems:

THEOREM 1. If $\Gamma$ is a lattice in an analytic group $G$, then $\Delta^{0}(\Gamma) \subset I(G)$.

THEOREM 2. If an analytic group $G$ contains a lattice, then the adjoint group $I(G)$ is closed in $A(G)$ (equivalently, in $A(\mathfrak{5})$ or in GL(5)).

In general, $I(G)$ is not a closed subgroup of $A(G)$ (see [7, pp. 127-128]). The converse of Theorem 2 is false, as we shall show at the end of this paper. The following proposition allows us to reduce the proofs of Theorems 1 and 2 to the case when $G$ is simply connected.

Proposition 1. Let $G$ be an analytic group, and let $G^{\prime}$ be the universal covering group of $G$; let ${ }^{*}$ denote either 1 or 2 . If Theorem * is true for $G^{\prime}$, then it is true for $G$.

Proof. Let $\Gamma$ be a lattice in $G$, let $\pi: G^{\prime} \rightarrow G$ be the covering map, and set $\pi^{-1}(\Gamma)=\Gamma^{\prime}$. Since $\Gamma^{\prime}$ is a lattice in $G^{\prime}$ and since $I(G)=I\left(G^{\prime}\right)$, Proposition 1 follows for ${ }^{*}=2$. Next, let $\Sigma\left(\Gamma^{\prime}\right)=\left\{\tau \in A\left(G^{\prime}\right) \mid \tau\left(\Gamma^{\prime}\right)=\Gamma^{\prime}\right\}$; then $\Sigma\left(\Gamma^{\prime}\right)$ is a closed subgroup of $A\left(G^{\prime}\right)$ such that $\Sigma\left(\Gamma^{\prime}\right) \supset \Delta\left(\Gamma^{\prime}\right)$; we denote the identity component of $\Sigma\left(\Gamma^{\prime}\right)$ by $\Sigma^{0}\left(\Gamma^{\prime}\right)$. For a fixed $\gamma \in \Gamma^{\prime}, \Sigma^{0}\left(\Gamma^{\prime}\right) \cdot \gamma$ is a connected subset of $\Gamma^{\prime}$, and hence is equal to $\gamma$. This proves that $\Sigma^{0}\left(\Gamma^{\prime}\right)=\Delta^{0}\left(\Gamma^{\prime}\right)$. Let $D$ be the kernel of $\pi$; $D$ is a discrete, central subgroup of $G^{\prime}$, and in the identification $A(G) \subset A\left(G^{\prime}\right)$,

$$
\Delta(\Gamma)=\left\{\tau \in A\left(G^{\prime}\right) \mid \tau(\gamma)=\gamma \bmod D, \text { for all } \gamma \in \Gamma^{\prime}\right\} \subset \Sigma\left(\Gamma^{\prime}\right),
$$

whence $\Delta^{0}(\Gamma) \subset \Sigma^{0}\left(\Gamma^{\prime}\right)=\Delta^{0}\left(\Gamma^{\prime}\right)$. Proposition 1 now follows for $*=1$. Q.E.D.

From now on we will assume that $G$ is a simply connected, analytic group.

The paper is organized into five sections. In $\$ 2$ we develop some necessary cohomological machinery, which we apply in $\S 3$ to obtain Theorem 1 when $G$ is solvable. In $\S 4$ we develop some machinery for passing to the general case. In $\S 5$ we complete the proof of Theorem 1 , and then, using the results of $\S 4$ and Theorem 1, we prove Theorem 2. Finally, in the appendix at the end of this paper, we show that the converse of Theorem 2 is false.

Professor H. C. Wang pointed out to us the construction of $R^{\prime}$ and $\Gamma_{2}$ on page 458 , and the possible usefulness of this construction for our purposes (his original construction was for uniform lattices). The appendix also derives from one of his suggestions. Our results were originally formulated for uniform lattices and the extension to lattices was suggested by Professor A. Borel. The first-named author became aware of the problem treated in Theorem 2, in a discussion with 
Professor J. A. Wolf. We are pleased to acknowledge our gratitude for all these valuable suggestions and discussions.

2. Cohomological tools. In this section we shall describe some results concerning the cohomological theory of Lie groups based on $C^{\infty}$-cochains (see [4] and [12]). Let $L$ be a Lie group and let $\rho: L \rightarrow \mathrm{GL}(V)$ be a continous representation of $L$ in the finite-dimensional, real vector space $V$. For a positive integer $q$, let $F^{g}(L, V)$ be the set of all $C^{\infty}$-maps of $L \times L \times \cdots \times L$ (q times) into $V$; let $F^{0}(L, V)=V$. For each integer $q \geqq 0$ we define a coboundary operator

by

$$
d^{q}: F^{q}(L, V) \rightarrow F^{q+1}(L, V)
$$

$$
d^{0} v(a)=v-\rho(a) v, \quad v \in V, \quad a \in L ;
$$

and for $q>0, f \in F^{q}(L, V)$

$$
\begin{aligned}
& d^{q} f\left(a_{1}, \cdots, a_{q+1}\right)=(-1)^{q+1}\left[\rho\left(a_{1}\right) f\left(a_{2}, \cdots, a_{q+1}\right)\right. \\
& \left.\quad+\sum_{i=1}^{q}(-1)^{i} f\left(a_{1}, \cdots, a_{i} a_{i+1}, \cdots, a_{q+1}\right)+(-1)^{q+1} f\left(a_{1}, \cdots, a_{q}\right)\right],
\end{aligned}
$$

for $a_{1}, \cdots, a_{q+1} \in L$. We set $Z^{q}(L, V)=\operatorname{kernel} d^{q}, B^{0}(L, V)=0$ and for $q \geqq 1$, $B^{q}(L, V)=$ image $d^{q-1}$. Finally, we set $H^{q}(L, V)=Z^{q}(L, V) / B^{q}(L, V) . Z^{q}(L, V)$ is called the space of $q$-cocycles, $B^{q}(L, V)$ the space of $q$-coboundaries, and $H^{q}(L, V)$ the $q$ th cohomology group of $L$ with respect to $\rho$. When $L$ is a discrete group, the $H^{q}(L, V)$ defined above, coincide with the usual Eilenberg-MacLane groups.

Let $M \subset L$ be a closed subgroup; then restricting $\rho$ to $M$, we obtain a continuous representation $\rho \mid M: M \rightarrow \mathrm{GL}(V)$, and with respect to $\rho \mid M$ we can form the complex $\left\{F^{q}(M, V)\right\}$ and the cohomology groups $H^{q}(M, V)$. For each $q \geqq 0$ we have the restriction map $\phi^{q}: F^{q}(L, V) \rightarrow F^{q}(M, V)$, and these maps commute with the coboundary operators; hence we have an induced map $\Phi^{q}: H^{q}(L, V)$ $\rightarrow H^{q}(M, V)$, called the $q$ th restriction homomorphism. When $q=1$, we will drop the superscript, and simply write $\phi$ and $\Phi$.

Proposition 2. Let $L$ be a Lie group, and $\Gamma$ a lattice in $L$; then the restriction homomorphism $\Phi: H^{1}(L, V) \rightarrow H^{1}(\Gamma, V)$ is one-one.

Proof. Let $f$ be in $Z^{1}(L, V)$ and let us assume that there exists $v \in V$ such that $f(\gamma)=v-\rho(\gamma) v$ for all $\gamma \in \Gamma$. Define $f^{\prime} \in Z^{1}(L, V)$ by $f^{\prime}(a)=f(a)-(v-\rho(a) v)$ for $a \in L$; then $f^{\prime}(\gamma)=0$ for all $\gamma \in \Gamma$. We wish to show that $f \in B^{1}(L, V)$. However, this is true if and only if $f^{\prime} \in B^{1}(L, V)$, so we can assume without loss of generality that $f(\gamma)=0$ for all $\gamma \in \Gamma$.

Since $f \in Z^{1}(L, V)$ we have from (1)

$$
f(a b)=f(a)+\rho(a) f(b), \quad a, b \in L .
$$


Hence for $\gamma \in \Gamma$, we obtain $f(a \gamma)=f(a)$. Thus, letting $a^{\prime}=a \Gamma \in L / \Gamma, f$ induces a map $f^{\prime}: L / \Gamma \rightarrow V$ such that $f^{\prime}\left(a^{\prime}\right)=f(a)$. Let $d a^{\prime}$ denote the invariant measure on $L / \Gamma$ with total volume equal to one. Set $w=\int_{L / \Gamma} f^{\prime}\left(a^{\prime}\right) d a^{\prime}$; then for $b \in L$

$$
\begin{aligned}
w-\rho(b) w & =\int_{L / \Gamma}\left[f^{\prime}\left(a^{\prime}\right)-\rho(b) f^{\prime}\left(a^{\prime}\right)\right] d a^{\prime} \\
& =\int_{L / \Gamma}\left[f^{\prime}\left(a^{\prime}\right)-\left(f^{\prime}\left(b a^{\prime}\right)-f^{\prime}\left(b^{\prime}\right)\right)\right] d a^{\prime} \\
& =f^{\prime}\left(b^{\prime}\right)=f(b),
\end{aligned}
$$

so that $f=d^{0} w \in B^{1}(L, V)$.

Q.E.D.

We denote the group of all continuous automorphisms of $L$ by $A(L)$. We let $\boldsymbol{R}$ denote the real line, and by a one-parameter group of automorphisms of $L$ we mean a $C^{\infty}$-map

$$
\tau: \boldsymbol{R} \times L \ni(t, a) \rightarrow \tau(t, a)=\tau_{t}(a) \in L,
$$

such that each $\tau_{t} \in A(L)$ and for all $s, t \in R, \tau_{t+s}=\tau_{t} \circ \tau_{s}$. For $a \in L$, we let $\tau^{\prime}(a)$ denote the tangent vector to the curve $\tau_{t}(a)$ at $t=0$. For $b \in L$, let $r_{b}$ and $l_{b}$ denote right and left translation by $b$, respectively. Since $\tau_{t}(a b)=\tau_{t}(a) \tau_{t}(b)$, we obtain

$$
\tau^{\prime}(a b)=d r_{b} \cdot \tau^{\prime}(a)+d l_{a} \cdot \tau^{\prime}(b)
$$

where $d r_{b}$ and $d l_{a}$ are the tangent linear maps induced by $r_{b}$ and $l_{a}$, respectively. $\tau^{\prime}$ defined above is thus a $C^{\infty}$-vector field on $L$, satisfying (3). Conversely, let us assume that $\xi$ is a $C^{\infty}$-vector field on $L$, satisfying (3) for $\tau^{\prime}=\xi$. Let $\tau_{t}(a)$ denote the unique integral curve to $\xi$ with $\tau_{0}(a)=a$. If $\tau_{t}(a)$ is defined for $t$ in the open interval $I_{\varepsilon}$ about 0 of radius $\varepsilon$, then for $t, s, t+s \in I_{\varepsilon}$, the uniqueness theorem for ordinary differential equations implies

$$
\tau_{t+s}(a)=\tau_{t}\left(\tau_{s}(a)\right)
$$

If $a$ and $b$ are in $L$, and if $\tau_{t}(a)$ and $\tau_{t}(b)$ are defined for $t \in I_{\varepsilon}$, then by computing the tangent vector to the curve $\tau_{t}(a) \tau_{t}(b)$ at $t_{0} \in I_{\varepsilon}$ and by using (3) and (4), we can see that $\tau_{t}(a) \tau_{t}(b)\left(t \in I_{\varepsilon}\right)$ is an integral curve of $\xi$; the uniqueness theorem of ordinary differential equations thus implies that $\tau_{t}(a b)$ exists for $t \in I_{\varepsilon}$, and in fact

$$
\tau_{t}(a b)=\tau_{t}(a) \tau_{t}(b)
$$

From the theory of ordinary differential equations we have that for $a_{0}$ in $L$ we can find a neighborbood $U$ of $a_{0}$ and $\varepsilon=\varepsilon\left(a_{0}\right)>0$, such that $\tau_{t}(a)$ is defined for all $t \in I_{\varepsilon}$ and $a \in U$, and the map $\tau: I_{\varepsilon} \times U \rightarrow L$, defined by $\tau(t, a)=\tau_{t}(a)$, is a $C^{\infty}$-map. Let us take $a_{0}=e$, the identity element of $L$; then $U$ generates $L^{0}$, the identity component of $L$, so that (5) implies that $\tau_{t}(a)$ is defined for all $a \in L^{0}$ and $t \in I_{\varepsilon}$. Now (4) implies that we can take $\varepsilon=\infty$. Next, using the fact that $\tau$ can be defined on $R \times L^{0}$ and applying (5), we can show that $\tau_{t}(a)$ can be 
defined for all $t \in R$ and all $a \in G$. Since $\tau_{t}$ and $\tau_{-t}$ are inverses, $\tau_{t}$ defines a oneparameter group of automorphisms, as defined above.

Let $\mathfrak{L}$ be the Lie algebra of $L$; that is, $\mathfrak{L}$ is the tangent space to $L$ at the identity. We then have that

$$
\tau_{t}(a)=\exp t X \cdot a \cdot \exp -t X, \quad t \in R, a \in L, X \in \mathfrak{L},
$$

defines a one-parameter group of automorphisms of $L$. Such a one-parameter group will be called a one-parameter group of inner automorphisms. A direct computation shows that for $\tau_{t}$ defined by (6)

$$
\tau^{\prime}(a)=d r_{a} \cdot X-d l_{a} \cdot X, \quad a \in L
$$

Thus we have

Proposition 3. Let $L$ be a Lie group and $\tau_{t}$ a one-parameter group of automorphisms of $L$; then the vector field $\tau^{\prime}$ satisfies (3), above. Conversely, a $C^{\infty}$ vector field $\tau^{\prime}$ on $L$ which satisfies (3), generates a one-parameter group of automorphisms. $\tau_{t}$ is a one-parameter group of inner automorphisms if and only if $\tau^{\prime}$ is given by (7) for some $X \in \mathfrak{L}$.

When the component group $L / L^{0}$ is finitely generated, $A(L)$, with the compactopen topology, is a Lie group (see [8]). In this case $a(L)$, the Lie algebra of $A(L)$, can be identified with $a^{*}(L)$, the Lie algebra of all $C^{\infty}$-vector fields on $L$, satisfying (3). We let $i^{*}(L)$ denote the subalgebra of $a^{*}(L)$, consisting of all those vector fields given by (7).

Let Ad denote the adjoint representation of $L$ in $\mathfrak{L}$, and form $Z^{1}(L, \mathfrak{L}), B^{1}(L, \mathfrak{L})$, and $H^{1}(L, \mathfrak{L})$ with respect to this representation. We define $\lambda: a^{*}(L) \rightarrow Z^{1}(L, \mathfrak{L})$ by

$$
\lambda(\xi)(a)=d r_{a^{-1}} \cdot \xi(a), \quad a \in L, \xi \in a^{*}(L) .
$$

(2) and (3) imply that $\lambda$ is well defined, and it is easily seen that $\lambda$ is linear, one-one, and onto. Moreover, from (7), we have that $\lambda\left(i^{*}(L)\right)=B^{1}(L, \mathfrak{L})$. We summarize these remarks in

Proposition 4. If we define $\lambda: a^{*}(L) \rightarrow Z^{1}(L, \mathfrak{L})$ by $(8), \lambda$ is a linear, onto isomorphism such that $\lambda\left(i^{*}(L)\right)=B^{1}(L, \mathfrak{L})$. Thus we have an induced, onto isomorphism $\Lambda: a^{*}(L) / i^{*}(L) \rightarrow H^{1}(L, \mathfrak{L})$.

3. Proof of Theorem 1 (solvable case). Let $G$ be a solvable (simply connected, analytic) group; let $\Gamma$ be a lattice in $G$. By a result of G. D. Mostow (see [13]), $\Gamma$ is a uniform lattice in $G$, and in particular, $\Gamma$ is finitely generated. Let $\mathfrak{N} \subset(\mathfrak{5}$ denote the nil-radical; that is, $\mathfrak{N}$ is the maximal nilpotent ideal in $\mathfrak{G}$. From [11] we know that $\Gamma \cap N=\Gamma_{N}$ is a lattice in $N$, and $\Gamma N$ is a closed subgroup of $G$, with identity component $N$. Since $\Gamma$ is finitely generated, $A(\Gamma N)$ is a Lie group. Let $A^{0}(G)$ and $A^{0}(\Gamma N)$ denote the identity components of $A(G)$ and $A(\Gamma N)$, respectively. For all $\delta \in a(\mathfrak{G}), \delta(\mathfrak{G}) \subset \mathfrak{N}(\operatorname{see}[9$, p. 51] $)$ so that $(\exp \delta)(g)=g \bmod N$ 
for $g \in G$. Thus we have a well-defined restriction homomorphism $\psi: A^{0}(G)$ $\rightarrow A^{0}(\Gamma N)$; let $\psi_{*}: a(G) \rightarrow a(\Gamma N)$ denote the corresponding Lie algebra homomorphism.

By Proposition 4 we have a one-one linear map $a(\Gamma N) \rightarrow Z^{1}(\Gamma N, \mathfrak{N})$. We compose this map with $Z^{1}(\Gamma N, \mathfrak{N}) \rightarrow Z^{1}(\Gamma, \mathfrak{N})$ (restriction map), and with $Z^{1}(\Gamma, \mathfrak{R}) \rightarrow Z^{1}(\Gamma, \mathfrak{F})$ (induced by the injection $\mathfrak{N} \rightarrow(\mathfrak{H})$, and we thus obtain a linear map $\mu: a(\Gamma N) \rightarrow Z^{1}(\Gamma,(5)$. It follows from [5] that $\mu$ is an onto isomorphism. We give the proof here that $\mu$ is one-one, which will suffice for our purposes. Thus if $\delta \in a(\Gamma N)$ and $\mu(\delta)=0$, then $\exp t \delta \cdot \gamma=\gamma$ for all $t \in \boldsymbol{R}$ and $\gamma \in \Gamma$. Since $\Gamma_{N}$ is a uniform lattice in $N$, we obtain, by applying A. I. Malcev's result in [10] to $N$ and $\Gamma_{N}$, that $\exp t \delta \cdot n=n$ for all $n \in N$; it follows that for all $t \in \boldsymbol{R}$, $\exp t \delta \in A^{0}(\Gamma N)$ is the identity. Thus $\delta=0$, so we have proved that $\mu$ is one-one.

Now the commutative diagram

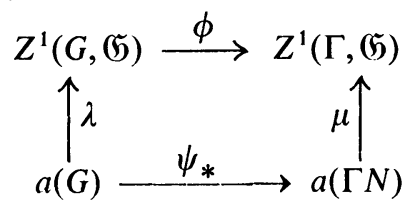

induces the following commutative diagram

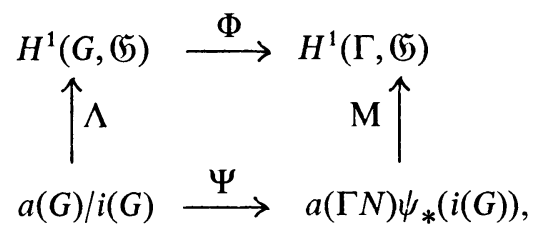

where $\mathrm{M}$ is induced by $\mu$ and $\Psi$ by $\psi_{*} . \Lambda$ is defined as before (see Proposition 4). We observe that since $G$ is connected, $i^{*}(G)$ corresponds to $i(G)=i(\mathfrak{b})$, under our identification of $a^{*}(G)$ with $a(G)$. Since $\mu\left(\psi_{*}(i(G))\right)=B^{1}(\Gamma,(\mathfrak{H})$, and since $\mu$ is one-one, it follows that $M$ is one-one. $\Lambda$ and $\Phi$ are one-one by Proposition 2 and Proposition 4. Thus $\Psi$ is one-one. Applying the result in [10], we obtain that $\Delta(\Gamma) \cap A^{0}(G)$ is the kernel of $\psi$ so that the Lie algebra of $\Delta^{0}(\Gamma)$ is the kernel of $\psi_{*}$. Since $\Psi$ is one-one, the kernel of $\psi_{*}$ is contained in $i(G)$, and hence $\Delta^{0}(\Gamma) \subset I(G)$. This concludes the proof of Theorem 1 when $G$ is solvable.

4. Tools for the general case. Propositions 5 and 6 below are proved in [3] (see Proposition 2.2 and the preceding remarks), but we present the proofs here for the sake of completeness. Let $\mathfrak{A} \subset \mathfrak{G}$ be the maximal semi-simple ideal, and let $\mathfrak{B}$ be the centralizer of $\mathfrak{U}$ in $\mathfrak{G}$; that is, $\mathfrak{B}=\{X \in \mathfrak{F} \mid[X, Y]=0$, for all $Y \in \mathfrak{U}\}$; then $\mathfrak{B}$ is an ideal of $\mathfrak{b}$, and since any derivation of a semisimple Lie algebra is inner, we have a direct sum decomposition $\mathfrak{G}=\mathfrak{A} \oplus \mathfrak{B}$. It is to be noted that both $\mathfrak{A}$ and $\mathfrak{B}$ are invariant by all automorphisms of $\mathfrak{G}$. Let $\mathfrak{R}$ be the (solvable) radical of $\mathfrak{G}$; then $\mathfrak{R} \subset \mathfrak{B}$, and $\mathfrak{B}$ has a Levi decomposition $B=\mathfrak{S} \oplus \mathfrak{R}$, where 
$\mathfrak{S}$ is a semi-simple subalgebra of $\mathfrak{B}$, but is not an ideal in $\mathfrak{B}$ unless $\mathfrak{S}=0$. $\mathfrak{G}=(\mathfrak{U} \oplus \mathfrak{S}) \oplus \mathfrak{R}$ gives a Levi decomposition of $\mathfrak{b}$.

Let 3 be the centralizer of $\Re$ in $\mathfrak{B}$; then 3 is an ideal of $\mathfrak{B}$ and so is $3+\Re$. Hence $3+\mathfrak{R} / \mathfrak{R}$ is a semi-simple ideal in $\mathfrak{B} / \mathfrak{R}$. Since $3+\mathfrak{R} / \mathfrak{R} \cong \mathbb{Z} / \mathfrak{Z} \cap \mathfrak{R}$, the Levi theorem implies that $\mathcal{Z}$ contains a semi-simple subalgebra $\mathfrak{D}$ such that $\mathcal{Z}=\mathfrak{D} \oplus(\mathfrak{Z} \cap \mathfrak{R})$. Since $\mathfrak{D}$ can always be imbedded in a Levi factor of $\mathfrak{G}$ (see $[9$, Corollary 1, p. 93] ), and since $\mathfrak{D}$ centralizes $\mathfrak{R}, \mathfrak{D}$ must be an ideal in $(\mathfrak{5}$. Thus $\mathfrak{D} \subset \mathfrak{A}$, and hence $\mathfrak{D} \subset \mathfrak{A} \cap \mathfrak{B}=0$; thus $\mathfrak{D}=0$ and $\mathfrak{Z} \subset \mathfrak{R}$. We note that $\mathfrak{R}$ and 3 are invariant under $A(\mathfrak{G})$, and that any ideal $\mathfrak{L}$ in $\mathfrak{G}$ containing $\mathfrak{R}$ is invariant under $A^{0}(\mathfrak{5})$, or equivalently under $a(\mathfrak{5})\left(A^{0}(\mathfrak{5})\right.$ is the identity component of $A(\mathfrak{5})$.)

Proposition 5. Let $\mathfrak{L}$ be an ideal of $\mathfrak{B}$ with $\mathfrak{R} \subset \mathfrak{L}$. Let $\delta$ be a derivation of $\mathfrak{B}$. If there exists an element $Y_{0} \in \mathfrak{L}$ such that

$$
\delta Y=\operatorname{Ad} Y_{0}(Y), \quad Y \in \mathfrak{L},
$$

then we can find $Y_{1} \in \mathfrak{L}$ such that $\delta=\operatorname{Ad} Y_{1}$ on $\mathfrak{B}$. In other words, let $f_{*}: a(\mathfrak{B}) \rightarrow a(\mathfrak{L})$ denote the restriction homomorphism; then $f_{*}^{-1}(i(\mathfrak{L}))=i_{\mathfrak{B}}(\mathfrak{L})$ $=\{\operatorname{Ad} Y \in i(\mathfrak{B}) \mid Y \in \mathfrak{L}\}$.

Proof. Since $\mathfrak{L} \supset \mathfrak{R}$, there exists a semisimple subalgebra $\mathfrak{E} \subset \mathfrak{B}$, such that we have a (vector space) direct sum decomposition $\mathfrak{B}=\mathbb{E} \oplus \mathfrak{L}$. We set $\delta_{1}=\delta-\operatorname{ad} Y_{0}$; then $\delta_{1}$ is a derivation of $\mathfrak{B}$ such that $\delta_{1} Y=0$ for $Y \in \mathfrak{L}$. For $X \in \mathbb{E}$ and $Y \in \mathfrak{L}$ we have $\left[\delta_{1} X, Y\right]=\delta_{1}[X, Y]-\left[X, \delta_{1} Y\right]=0.3_{1}$, the centralizer of $\mathfrak{L}$ in $\mathfrak{B}$, is an ideal satisfying $3_{1} \subset 3 \subset \mathfrak{R}$. The previous computation shows that for $X \in \mathfrak{E}$, we have $\delta_{1} X \in \mathfrak{Z}_{1}$. Thus $\delta_{1}$ induces a map from $\mathfrak{E}$ to $\mathfrak{Z}_{1}$, still denoted by $\delta_{1}$, such that

$$
\delta_{1}\left[X_{1}, X_{2}\right]=\left[\delta_{1} X_{1}, X_{2}\right]+\left[X_{1}, \delta_{1} X_{2}\right], \quad X_{1}, X_{2} \in \mathbb{E} .
$$

For $X \in \mathbb{E}$, let $\phi(X)$ denote the restriction of ad $X$ to $3_{1}$; then $\phi$ is a representation of $\mathfrak{E}$, and (1) implies that $\delta_{1}$ is a one-cocycle of $\mathbb{E}$ with respect to $\phi$. By the first Whitehead lemma, there exists $Y^{\prime} \in \mathcal{Z}_{1}$ such that $\delta_{1} X=\left[Y^{\prime}, X\right]$ for all $X \in \mathbb{E}$. Since $\delta_{1}=\operatorname{ad} Y^{\prime}=0$ on $\mathfrak{L}$, we have $\delta_{1}=\operatorname{ad} Y^{\prime}$ on $\mathfrak{B}$. Hence $\delta=\operatorname{ad}\left(Y_{0}+Y^{\prime}\right)$ on $\mathfrak{B}$ and we can take $Y_{1}=Y_{0}+Y^{\prime}$.

Q.E.D.

Proposition 6. Let $\mathfrak{M}$ be an ideal in $(\mathfrak{G}$ containing $\mathfrak{R}$. If $I(\mathfrak{M})$ is closed in $\mathrm{GL}(\mathfrak{M})$, then $\mathrm{I}(\mathfrak{5})$ is closed in GL((5)).

For the proof of Proposition 6, we utilize the following Lemma from [6]:

LEMMA 1. Let $H$ be an analytic subgroup of the general linear group $\mathrm{GL}(n, C)$. If the radical of $H$ is closed in $\mathrm{GL}(n, C)_{2}$ then so is $H$.

Proof of Proposition 6. $\mathfrak{M}$ has a direct sum decomposition $\mathfrak{M}=(\mathfrak{A} \cap \mathfrak{M}) \oplus \mathfrak{L}$, where $\mathfrak{L}$ is an ideal in $\mathfrak{B}$ and $\mathfrak{A} \cap \mathfrak{M}$ is semisimple. $I(\mathfrak{M})$ is the direct product of $I(\mathfrak{A} \cap \mathfrak{M})$ and $I(\mathfrak{L})$, so that if $I(\mathfrak{M})$ is closed, then $I(\mathfrak{L})$ is closed. 
Let $f: A^{0}(\mathfrak{B}) \rightarrow A^{0}(\mathfrak{L})$ denote the restriction homomorphism and let $f_{*}: a(\mathfrak{B}) \rightarrow a(\mathfrak{L})$ denote the corresponding Lie algebra homomorphism. Since $I(\mathfrak{L})$ is a closed subgroup of $A^{0}(\mathfrak{L})$, we have that $f^{-1}(I(\mathfrak{L})$ ) is a closed subgroup of $A^{0}(\mathfrak{B})$. Hence the analytic subgroup of $A^{0}(\mathfrak{B})$ corresponding to $f_{*}^{-1}(i(\mathfrak{L})) \subset a(\mathfrak{B})$ is a closed subgroup; however, Proposition 5 implies that $f_{*}^{-1}(i(\mathfrak{L}))=i_{\mathfrak{B}}(\mathfrak{L})$. The radical of $i_{\mathfrak{B}}(\mathfrak{L})$ is $i_{\mathfrak{B}}(\mathfrak{R})$. Since $i_{\mathfrak{B}}(\mathfrak{R})$ is also the radical of $i(\mathfrak{B})$, Lemma 1 implies that $I(\mathfrak{B})$ is a closed subgroup of $A(\mathfrak{B})$ (and hence of $G L(\mathfrak{B})$ ).

Now $\mathfrak{G}=\mathfrak{U} \oplus \mathfrak{B}$ with $[\mathfrak{U}, \mathfrak{B}]=0$; hence $I(\mathfrak{b})$ is the direct product of $I(\mathfrak{A})$ and $I(\mathfrak{B}) . I(\mathfrak{A})$ is closed in $\operatorname{GL}(\mathfrak{A})$ since $\mathfrak{U}$ is semisimple; also $I(\mathfrak{B})$ is closed in $\mathrm{GL}(\mathfrak{B})$ by our above argument. Hence $I(\mathfrak{G})$ is closed in $\mathrm{GL}(\mathfrak{5}) . \quad$ Q.E.D.

We now summarize some measure theoretic results which we will need. For more details see $[15, \S 9]$ or $[7$, Chapter 10$]$. If $S$ is a locally compact Hausdorff space and if $C_{c}(S)$ is the space of real valued, continuous functions on $S$ with compact support, then a positive measure on $S$ is a real valued linear functional $\mu: C_{c}(S) \rightarrow R$, such that if $f \in C_{c}(S)$ is nonnegative, then $\mu(f) \geqq 0$. If $P$ and $Q$ are locally compact, unimodular groups, such that $Q$ is a closed subgroup of $P$, then $P / Q$ has a left invariant measure which is unique up to a constant factor.

Proposition 7. Let $P$ be a locally compact topological group and let $Q$ and $R$ be closed subgroups of $P$ such that $Q \supset R$. If $P, Q$, and $R$ are all unimodular, then there exist invariant measures $\lambda, \mu, v$ on $P / Q, Q / R$, and $P / R$, respectively, such that for any function $f \in C_{c}(P / R)$ :

$$
\int_{P / R} f(p R) d v(p R)=\int_{P / Q}\left(\int_{Q / R} f(p q R) d \mu(q R)\right) d \lambda(p Q) .
$$

Proof. One simply has to verify (2) for $f \in C_{c}(P / R)$. But for such $f$ one can define $v(f)$ by the right hand side of (2), where one chooses the invariant measures $\mu$ and $\lambda$ arbitrarily. One sees immediately that $v$ is invariant, and hence $\lambda, \mu$, and $v$, chosen as above, satisfy (2).

Q.E.D.

Corollary. Assume $P, Q, R, \lambda, \mu$, and $v$ are as in Proposition 7; moreover, assume that $P$ is a Lie group and $v(P / R)<\infty$; then $\mu(Q / R)<\infty$ and $\lambda(P / Q)<\infty$.

Proof. It suffices to show that (2) holds when $f$ is identically equal to one. But then, since $P$ is a Lie group, we can find an increasing sequence of nonnegative functions $f_{n}$ in $C_{c}(P / R)$, which approach $f$ pointwise. The desired conclusion now follows from Proposition 7, and from the monotone convergence theorem.

Q.E.D.

Next we recall some results from [14]. If $P$ is a topological group and $H$ a subgroup of $P$, then $H$ is said to have the Selberg property (or property (S)) in case, for any open neighborhood $U$ of the identity and for any $p \in P$, there exists a positive integer $n$ with $p^{n} \in U H U$. If $f: P \rightarrow P^{\prime}$ is a continuous epimorphism, then $f(H)$ has property (S). A lattice $\Gamma$ in a Lie group $L$ has property (S) (see [1]). 
We retain the notation introduced at the beginning of this section. Let $\Omega$ and $\boldsymbol{R}^{\prime}$ be the maximal compact ideals in $\mathfrak{A}$ and $\mathfrak{S}$, respectively; then set $\mathfrak{C}=\mathfrak{R}+\boldsymbol{\Omega}^{\prime}$. $C$ is then the maximal analytic, compact, normal subgroup of a semi-simple part of $G$.

Lemma 2 [14, Theorem B, P. 211]. Let $\theta: G \rightarrow G / C R$ denote the projection. Suppose that $L$ is a closed subgroup of $G$ with its identity component solvable. If $\theta(L)$ has the property $(S)$, then $\theta(L)$ is discrete.

Lemma 3 [14, Theorem A, P. 211]. Let $P$ be an analytic group with simply connected radical $R$; let $\pi: P \rightarrow P / R$ be the projection; and let $L$ be a closed subgroup of $P$. If the identity component of $L$ is solvable, then the identity component of the closure of $\pi(L)$ is also solvable.

Proposition 8. Let $\Gamma \subset G$ be a lattice; then $\Gamma C R$ is a closed subgroup of $G$ with identity component $C R$.

Proof. $\Gamma$ has property (S) in $G$, and hence $\theta(\Gamma)$ has property $(\mathrm{S})$ in $G / C R$. Hence Lemma 2 implies $\theta(\Gamma)$ is discrete. Proposition 8 now follows. Q.E.D.

\section{Proofs of Theorems 1 and 2.}

(i) Proof of Theorem 1. Let $\Gamma$ be a lattice in $G$; then $G$ is unimodular and hence if $\mathfrak{I}$ is any ideal in $(\mathfrak{b}$ such that $\mathfrak{R} \subset \mathfrak{I}$, then $\operatorname{det}(\operatorname{Ad} g \mid \mathfrak{I})=1$ for any $g \in G$ (where $\operatorname{Ad} g \mid \mathfrak{I}$ denotes the restriction of $\operatorname{Ad} g$ to $\mathfrak{I}$ ). In particular $I$ is unimodular. By Proposition $8, \Gamma C R$ is a closed subgroup with identity component $C R$. By our previous remark $\Gamma C R$ is thus a unimodular, closed subgroup of $G$. Hence we can apply the Corollary to Proposition 7 to $G \supset \Gamma C R \supset \Gamma$. We obtain that $\Gamma$ is a lattice in $\Gamma C R$. Hence $\Gamma_{1}=\Gamma \cap C R$ is a lattice in $C R$.

Let $\pi: C R \rightarrow C=C R / R$ be the natural projection, and let $T$ be the closure of $\pi\left(\Gamma_{1}\right)$. By Lemma 3, we show that $T^{0}$, the identity component of $T$, is solvable. Since $C$ is compact and $T$ is closed, we must have that $T^{0}$ is a torus of finite index in $T$. We set $R^{\prime}=T^{0} R$; then $R^{\prime}$ is a closed, solvable, analytic subgroup of $C R$. We set $\Gamma_{2}=\Gamma_{1} \cap R^{\prime}$; then

$$
\Gamma_{1} / \Gamma_{2} \cong \Gamma_{1} R^{\prime} / R^{\prime}=\pi\left(\Gamma_{1}\right) T^{0} R / T^{0} R \subset T R / T^{0} R \cong T / T^{0},
$$

so that $\Gamma_{2}$ is of finite index in $\Gamma_{1}$. Thus $\Gamma_{2}$ is a lattice in $C R$. By our observation at the beginning of this section, $R$ is unimodular. It then follows easily that $R^{\prime}=T^{0} R$ is unimodular; hence, applying the Corollary to Proposition 7 to $C R \supset R^{\prime} \supset \Gamma_{2}$, we find that $\Gamma_{2}$ is a lattice in $R^{\prime}$. Hence, by G. D. Mostow's result in [13], $\Gamma_{2}$ is a uniform lattice in $R^{\prime}$, and hence in $C R$. Since $\Gamma_{2} \subset \Gamma_{1}$, we have that $\Gamma_{1}$ is a uniform lattice in $C R$. Thus we have proved

Proposition 9. Let $\Gamma$ be a lattice in $G$; then $\Gamma_{1}=\Gamma \cap C R$ is a uniform lattice in $C R$. 
Let $\tau$ be in $\Delta(\Gamma)$. Since the closure of $\Gamma_{2} R$ is $T^{0} R=R^{\prime}, \tau$ maps $R^{\prime}$ onto itself; hence we have the restriction homomorphism $\omega: \Delta(\Gamma) \rightarrow A\left(R^{\prime}\right)$. Using Theorem 1 (solvable case) we have that $\omega\left(\Delta^{0}(\Gamma)\right) \subset I\left(R^{\prime}\right)$. Now we choose neighborhoods $U \subset I\left(R^{\prime}\right)$ and $V \subset R^{\prime}$, of the identity such that we have a local cross-section $\psi: U \rightarrow V$ of the homomorphism $\mathrm{Ad}: R^{\prime} \rightarrow I\left(R^{\prime}\right)$; that is, $\operatorname{Ad} \circ \psi=$ identity. Next choose a connected neighborhood $W$ of the identity in $\Delta^{0}(\Gamma)$ such that $\omega(W) \subset U$. For $\sigma \in W$, we define $\lambda(\sigma)$ to be $\operatorname{Ad}(\psi(\omega(\sigma))) \circ \sigma^{-1}$, where Ad here denotes the adjoint map $\operatorname{Ad}: G \rightarrow I(\mathfrak{G})$; then $\lambda(\sigma)$ is an automorphism of $G$, whose restriction to $R^{\prime}$, and a fortiori to $R$, is the identity. We denote the restriction of $\lambda(\sigma)$ to $B$ by $\mu(\sigma) . \mu: W \rightarrow A(B)$ is continuous and $W$ is connected. Hence $\mu(W)$ is connected. Since the restriction of $\mu(\sigma), \sigma \in W$, to $R$ is the identity, Proposition 5 implies that $\mu(\sigma) \in I(B)$. On the other hand, $\lambda(W)$ being connected, the restriction of $\lambda(W)$ to semi-simple, normal subgroup $A$ is composed of inner automorphisms. Hence $\lambda(W) \subset I(G)$, and hence $W \subset I(G)$. Since $W$ generates $\Delta^{0}(\Gamma)$, we have that $\Delta^{0}(\Gamma) \subset I(G)$.

Q.E.D.

(ii) Proof of Theorem 2 under the additional assumption that $\Gamma$ is a uniform lattice. Let $\operatorname{Ad}: G \rightarrow I(G)$ denote the adjoint map, and let $\sigma_{1}=\operatorname{Ad} \gamma_{1}, \sigma_{2}=\operatorname{Ad} \gamma_{2}, \cdots$, be a sequence of inner automorphisms where $\gamma_{n} \in \Gamma, n=1,2, \cdots$. Suppose that the sequence $\sigma_{n}$ approaches $\sigma \in A(G)$; also, let $\gamma^{(1)}, \cdots, \gamma^{(s)}$ be a finite system of generators for $\Gamma$; then for each $i=1,2, \cdots, s$ we can find a positive integer $N(i)$ such that $\sigma^{-1} \sigma_{n}\left(\gamma^{(i)}\right)=\gamma^{(i)}$ for $n>N(i)$. Next, since $\Delta(\Gamma)$ is closed in $A(G)$, we can find an open neighborhood $U$ of the identity in $A(G)$ such that

$$
\Delta(\Gamma) \cap U \subset \Delta^{0}(\Gamma) \subset I(G) .
$$

We can find a positive integer $M$ such that for $n>M, \sigma^{-1} \sigma_{n} \in U$. Let $n$ be an integer larger than $M$ and all the $N(i)$; then $\sigma^{-1} \sigma_{n} \in \Delta(\Gamma) \cap U \subset I(G)$, so that $\sigma \in I(G)$; thus we have proved that $\operatorname{Ad}(\Gamma)^{-}$, the closure of $\operatorname{Ad}(\Gamma)$ in $\operatorname{GL}(\mathfrak{G})$, is contained in $I(G)$. Let $S$ denote the coset space $I(G) / \operatorname{Ad}(\Gamma)^{-}$. Since Ad induces a continuous map of the compact space $G / \Gamma$ onto $S, S$ is compact, and hence closed in $A(G) / \operatorname{Ad}(\Gamma)^{-}$; it follows that $I(G)$ is closed in $A(G)$ Q.E.D.

(iii) Proof of Theorem 2. By Proposition $9, \Gamma_{1}$ is a uniform lattice in $C R$ and hence, by (ii), $I(C R)$ is closed in $A(C R)$. Hence Proposition 6 implies that $I(G)$ is closed in $A(G)$.

APPENDIX. We will now give an example of a solvable, unimodular, analytic group $G$, such that $I(G)$ is closed in GL(F), but $G$ does not contain a lattice.

For every triple of real numbers $\alpha=\left(\alpha_{1}, \alpha_{2}, \alpha_{3}\right) \neq(0,0,0)$, such that $\alpha_{1}+\alpha_{2}+\alpha_{3}=0$, let $A_{\alpha}$ be the one-parameter matrix group

$$
\left\{B_{t}=\exp t\left[\begin{array}{lll}
\alpha_{1} & 0 & 0 \\
0 & \alpha_{2} & 0 \\
0 & 0 & \alpha_{3}
\end{array}\right] \mid t \in R\right\} .
$$


Let $G_{\alpha}$ be the semidirect product of $A_{\alpha}$ and $\boldsymbol{R}^{3} . N$, the nil-radical of $G_{\alpha}$, is $\boldsymbol{R}^{3}$. Assume $G_{\alpha}$ contains a lattice $\Gamma$; then $\Gamma$ is a uniform lattice, since $G_{\alpha}$ is solvable (see [13]). $\Gamma \cap N=\Gamma_{N}$ is a lattice in $N$ and $\Gamma N / N$ is a lattice in $G_{\alpha} / N=A_{\alpha}$ (see [11]). Since $\Gamma_{N}$ is just the integral span of an appropriate basis in $N$, and since the linear transformations in the lattice $\Gamma N / N \subset A_{\alpha}$ leave $\Gamma_{N}$ invariant, we have that the matrices representing $\Gamma N / N$, with respect to a suitable basis in $N$, are all integral. Hence for some $t \neq 0$, all the coefficients of the characteristic polynomial of $B_{t}$ are integral. Let $J$ denote the set of all positive real numbers satisfying an equation of the form $X^{3}+a_{1} X^{2}+a_{2} X-1=0$, where $a_{1}$ and $a_{2}$ are integers. Let $J^{\prime}=\{\log j \mid j \in J\}$; then $J^{\prime 3}=J^{\prime} \times J^{\prime} \times J^{\prime}$ is a countable subset of $R^{3}$. Hence we can find $\alpha=\left(\alpha_{1}, \alpha_{2}, \alpha_{3}\right)$ with $\alpha_{1}+\alpha_{2}+\alpha_{3}=0$, such that $t \alpha \in J^{\prime 3}$ implies $t=0$. For such $\alpha, G_{\alpha}$ cannot contain a lattice. On the other hand, $G$ is unimodular and $I\left(G_{\alpha}\right)$ is closed in $A\left(G_{\alpha}\right)$, for all $\alpha$ as above.

\section{BIBLIOGRAPHY}

1. A. Borel, Density properties for certain subgroups of semi-simple groups without compact components, Ann. of Math. (2) 72 (1960), 179-188.

2. C. Chevalley, Theory of Lie groups. I, Princeton Math. Series 8, Princeton Univ. Press, Princeton, N. J., 1946.

3. W. T. van Est, Some theorems on (CA) Lie algebras. II, Nederl. Akad. Wetensch. Proc. Ser. A 55 (1952), 558-568.

4. - On the algebraic cohomology concepts in Lie groups. I, II, Nederl. Akad. Wetensch. Proc. Ser. A 58 (1955), 225-233; 286-294.

5. H. Garland, On the cohomology of lattices in solvable Lie groups, Ann. of Math. 84 (1966), 174-195.

6. M. Goto, Faithful representations of Lie groups. I, Math. Japon. 1 (1948), 107-119.

7. S. Helgason, Differential geometry and symmetric spaces, Pure and Applied Math. 12, Academic Press, New York, 1962.

8. G. Hochschild, The automorphism group of a Lie group, Trans. Amer. Math. Soc. 72 (1952), 209-216.

9. N. Jacobson, Lie algebras, Tracts in Math. 10, Interscience, New York, 1962.

10. A. I. Malcev, On a class of homogeneous spaces, Amer. Math. Soc. Transl. No. 39 (1951), $33 \mathrm{pp}$.

11. G. D. Mostow, Factor spaces of solvable groups, Ann. of Math. 60 (1954), 1-27.

12. — Cohomology of topological groups and solvmanifolds, Ann. of Math. 73 (1961), 20-48.

13. —_ Homogeneous spaces with finite invariant measure, Ann. of Math. (2) 75 (1962), 17-37.

14. H. C. Wang, On the deformation of lattice in a Lie group, Amer. J. Math. 85 (1963), 189-212.

15. A.Weil,L'intégration dansles groupes topologiques et ses applications, Hermann, Paris, 1940.

YALE UNIVERSITY,

New Haven, Connecticut

The Institute for Advanced Study,

Princeton, NeW Jersey

The University of Pensylvania,

Philadel phia, Pennsylvania 